\title{
The Fluorescent Measurement in D. Magna Feeding Suppression Bioassay during the Pre-exposure in Potassium Dichromate for 1, 2, 3, 4 and 5 Hours
}

\author{
Kamaya Minori, Ginatullina Elena, Yamagata Kohei
}

\begin{abstract}
The present study focused on observation of effect of potassium dichromate as standard toxicant on feeding activity of D. magna. Isolated feeding suppression was measured as fluorescence intensity of ingested microbeads by daphnids and expressed as its feeding rate (EC50FI). We calculated the sub-lethal feeding suppression endpoints for 1, 2, 3, 4 and 5 hours exposure in potassium dichromate and compared these values with $E_{50}$ concentration of immobilization test of $D$. magna. The most close to immobilization (EC50) result was obtained as for 4 as well for 5 hours exposure time; however 4 hs exposure was chosen as more adequate exposure time for our design of feeding suppression bioassay.
\end{abstract}

Key words: acute test, feeding rate, fluorescent intensity, polyester microbeads.

\section{INTRODUCTION}

Among the methods of biological testing the determination of the environment toxicity using lower crustaceans plays an important role, primarily using Daphnia magna Straus, 1820. The methods of daphnia biological testing are widely used for the environmental monitoring around of the world. The crustaceans mortality is mainly used as a test reaction, and the observations of fertility and offspring quality changes are performed when the chronic toxic effects are revealed [Tarazona, 2005; Braginskii, 2000].

At the present stage of technological development, the sensitive determination of toxic substances presence in the water is possible according to the behavioral characteristics of test objects, by the means, of their feeding activity; in with evaluation toxicity is possible using sub-lethal endpoint that found to be more sensitive than EC ${ }_{50}$ of $D$. magna lethality acute test.

Revised Manuscript Received on February 07, 2020.

* Correspondence Author

Kamaya Minori*, Department of Applied Chemistry, Kogakuin University, Tokyo, Japan. Email: bt74290@ns.kogakuin.ac.jp

Ginatullina Elena, Department of Ichtyology and Hydrobiology, Institute of Zoology Uz Academy of Sciences, Tashkent, Uzbekistan. Email: e-ginatullina@yandex.ru

Yamagata Kohei, Department of Applied Chemistry, Kogakuin University, Tokyo, Japan. Email: bm18049@ns.kogakuin.ac.jp

(C) The Authors. Published by Blue Eyes Intelligence Engineering and Sciences Publication (BEIESP). This is an open access article under the CC BY-NC-ND license (http://creativecommons.org/licenses/by-nc-nd/4.0/)
Lürling etal. (2011) found that the feeding rate of D. magna using artificial beads is a more sensitive endpoint than an acute lethal test. Alterations in the feeding and digestive behaviour of daphnids as endpoints were compared by De Coen and Janssen (1998) and De Coen et al. (1998), where ingestion rate was assessed using fluorescent microbeads and measuring fluorescence. In these studies, a clear inhibition of the ingestion activity was experienced, while the digestive enzyme activity proved to be less affected. The inhibition of feeding has been applied by several authors to assess the possible ecotoxicological effects of different contaminants, such as metals (Ferrando and Andreu, 1993) and pesticides (Fernandez-Cassalderrey et al., 1994).

In the past, our team has been developed a design of $D$. magna feeding suppression assay through the ingesting by daphnids and further fluorescence measurement the polyester fluorescent micro beads. In this paper we propose a short-term D. magna feeding suppression test as a cost effective and ecological relevant sub-lethal bioassay. We have obtained an acute sub-lethal endpoint $\left(\mathrm{EC}_{50 \mathrm{FI}}\right)$ in feeding test and compare sensitivity of the test with standard $24 \mathrm{~h}$ immobility/lethality test endpoint $\left(\mathrm{EC}_{50}\right)$ using potassium dichromate as the reference toxicant.

\section{METHODS}

\section{A. Daphnia magna culture.}

D. magna was cultivated in an incubator at $21{ }^{\circ} \mathrm{C}$ using Elendt M4 culture water according to standard procedures [USEPA, 1985] and fed daily with Spirullina' powder.

\section{B. 24-hour immobilization bioassay EC 5 .}

We used 20 neonates of D. magna not older than $24 \mathrm{~h}$. Procedure and calculation of $\mathrm{EC}_{50}$ using the conventional 24 $\mathrm{h}$ bioassay with a lethal endpoint was carried out according to standard procedure [USEPA, 1985; J.I.S., 1992]. Twenty neonates were placed into each of $20 \mathrm{~mL}$ glass beaker filled with $5 \mathrm{~mL}$ of toxicant (5 different diluted toxicant solutions) and into one beaker filled with $20 \mathrm{ml}$ of control water (Fig.1). 
The Fluorescent Measurement in D. magna feeding suppression bioassay during the pre-exposure in potassium dichromate for 1, 2, 3,4 and 5 hours

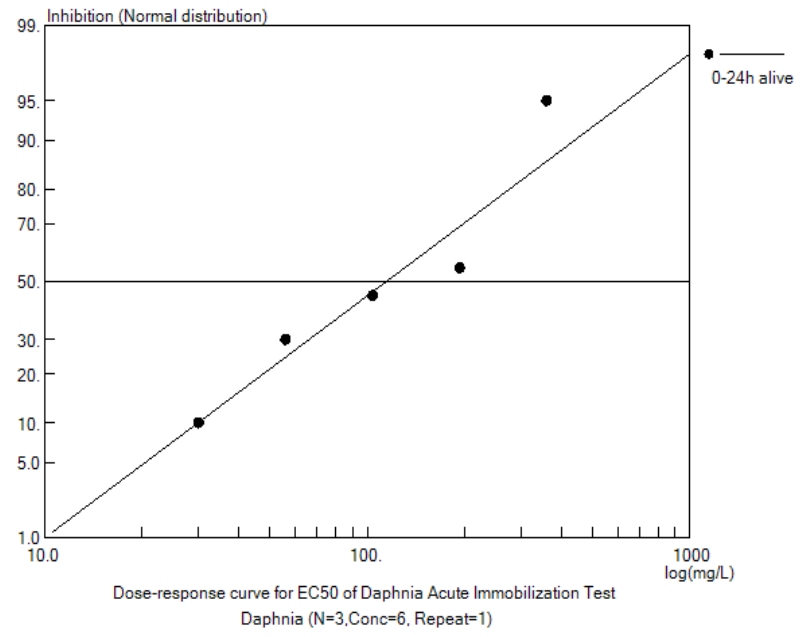

Fig. 1 Relation between percent of immobilized daphnids and logarithm of $\mathrm{K}_{2} \mathrm{Cr}_{2} \mathrm{O}_{7}(\mathrm{mg} / \mathrm{l})$ after $24 \mathrm{hs}$

\section{Design of feeding suppression bioassay}

The feeding ability of daphnids, which suppressed by influencing of toxicant, we measured with help of fluorescent intensity instrument (Schimadzu fluorometr). We determined that optimal fluorescence occurred by wavelengths: $E x=365$ $\mathrm{nm}, \mathrm{Em}=413 \mathrm{~nm}$ with microbeads concentration of $5 \times 10^{9}$ may be observed if microbeads add to toxicant solution 20 minutes before of completion of toxicant exposure. Parameters of fluorescence intensity measurement are in Tab. 1.

Table 1 Parameters of fluorescent measurement

\begin{tabular}{|l|l|}
\hline Excitation, $\lambda$ & $365 \mathrm{~nm}$ \\
\hline Emission, $\lambda$ & $415 \mathrm{~nm}$ \\
\hline Volume of detector cell,ml & $1.2 \mathrm{ml}$ \\
\hline Volume of test solution, $\mathrm{ml}$ & $10 \mathrm{ml}$ \\
\hline Total volume of test solution & $10 \mathrm{ml}+100 \mu \mathrm{L}$ \\
\hline Amount and final concentration of & $100 \mu \mathrm{L}$ of \\
microbeads in toxicant solution & $\mathrm{d}=0.2 \mu \mathrm{m}$ \\
& $5.00 \times 10^{9}$ beads/ \\
& $\mathrm{mL}$ \\
\hline
\end{tabular}

Mature daphnids was taken from incubator the day before experiment and put into dilution water. In the day of experiment was prepared the beakers with $10 \mathrm{ml}$ of 5 different concentrations of toxicant and one control beaker, and 24 h old $D$. magna neonates were separated from mature culture.

In the first experimental sequence 10 daphnids was exposed into both: toxicant solutions and control water used $20 \mathrm{ml}$ glass beakers. In the second experimental sequence (blank experiment) we used filled with the identical to first sequence toxicant concentrations and control water beakers, but without 10 daphnids in each beaker. For each exposure period (1, 2, 3, 4 and 5 hours) the same amount of microbeads $100 \mu \mathrm{L}\left(4.55 \times 10^{12}\right)$ was added 20 min before of completion of exposure. After exposure, daphnids was removed, and the fluorescent intensity measurement of toxicant solution and control water was done.

\section{Calculation of sub-lethal feeding endpoint (EC $50 \mathrm{FI})$}

To calculate $\mathrm{EC}_{50}$ endpoint due to suppression of $D$. magna feeding we have done the procedure of fluorescent intensity measurement for two sequences of experiment carried out one after another with a short period of time. First experimental sequence measurement of fluorescent intensity (with 10 daphnids) was defined as $\mathrm{FI}_{\text {expmt, the blank }}$ measurement of fluorescent intensity (without 10 daphnids) -

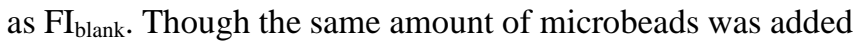
in to each "blank" glass beaker filled with control solution or 5 toxicant solutions, the values of $\mathrm{FI}_{\text {blank }}$ was different for each of 5 toxicant concentration.

Due to toxicant exposure daphnids filtration ability declines, and fluorescent intensity increasing proportional to remained amount of fluorescent microbeads. The relative feeding rate of daphnids was expressed as equation (\%): toxicant solution $\left(\mathrm{FI}_{\text {blank }}{ }^{-} \mathrm{FI}_{\text {expmt }}\right) /$ control water $\left(\mathrm{FI}_{\text {blank- }}\right.$ $\mathrm{FI}_{\text {expmt }}$ x100 (eq.).

The EC $_{50 \mathrm{FI}}$ concentration was taken as concentration for what it was observed of $50 \%$ of feeding rate (half of control feeding rate). Using the relation between relative feeding rate (\%) and log of toxicant concentration we calculated the EC $_{50 \mathrm{FI}}$ endpoints (Fig.2).

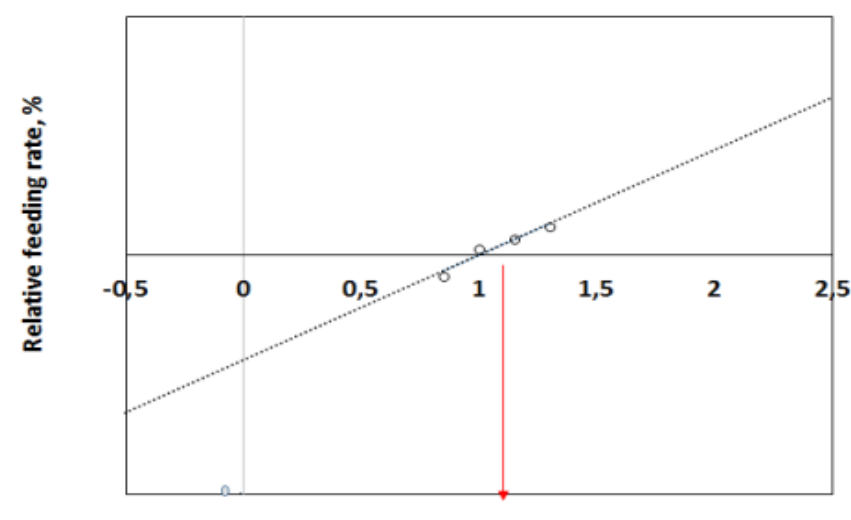

Log of potassium dichromate concentration

Fig. 2 Relation between feeding rate and $\log$ of $\mathrm{K}_{2} \mathrm{Cr}_{2} \mathrm{O}_{7}$ (mg/l) after $1 \mathrm{~h}$ exposure

For each toxicant and each exposure time three replicates of test was done.

\section{E. Toxicant and fluorescent microbead solutions.}

A stock solution of potassium dichromate $(1000 \mathrm{mg} / \mathrm{L}$; Kanto Kagaku Co., Ltd.) was prepared by using MilliQ water (Organo Corporation). In Tab.2 shown potassium dichromate concentration used for lethality and feeding experiments.

Table 2 The ranges of concentration used for 1, 2, 3,4, 5 hours feeding and $24 \mathrm{~h}$ lethality tests

\begin{tabular}{|l|l|}
\hline $\begin{array}{l}\text { Exposure time in } \\
\text { toxicant solution }\end{array}$ & $\begin{array}{c}\text { Toxicant' range of } \\
\mathrm{K}_{2} \mathrm{Cr}_{2} \mathrm{O}_{7} \text { concentrations, } \mathrm{mg} / \mathrm{l}\end{array}$ \\
\hline $1 \mathrm{~h}$ microbeads & $1.0 ; 1.41 ; 2.0 ; 2.83 ; 4.0$ \\
\hline $2 \mathrm{~h}$ & $0.8 ; 1.17 ; 1.7 ; 2.47 ; 3.6$ \\
\hline $3 \mathrm{~h}$ & $0.6 ; 0.91 ; 1.39 ; 2.11 ; 3.2$ \\
\hline $4 \mathrm{~h}$ & $0.5 ; 0.7 ; 1.1 ; 1.7 ; 2.5$ \\
\hline $5 \mathrm{~h}$ & $0.5 ; 0.8 ; 1.1 ; 1.7 ; 2.5$ \\
\hline $24 \mathrm{~h}$ & $0.5 ; 0.8 ; 1.1 ; 1.7 ; 2.5$ \\
\hline
\end{tabular}

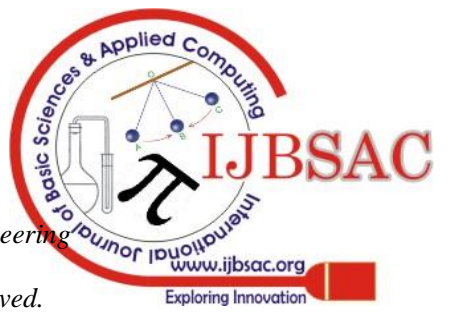


Stock solution of fluorescent microbead was prepared by suspending $2 \%$ FluoSphere carboxylate modified microspheres F8805 ( $\mathrm{d}=0.2 \mu \mathrm{m}$; invitrogen.com) in MilliQ water (Organo Corporation) to a concentration of $4.55 \times 10^{12}$ particles per $\mathrm{mL}$ was diluted in 910 times and used as the final concentration in toxicant solution (total amount in test solution of $20 \mathrm{~mL}$ was $5.00 \times 10^{7}$ particles $/ \mathrm{mL}$. For experiments treatment as both: toxic substances dilution and control water was handled a following solution: $\mathrm{CaCl}_{2} \cdot 2 \mathrm{H}_{2} \mathrm{O}$ -293.8mg/L, $\mathrm{MgSO}_{4} \cdot 7 \mathrm{H}_{2} \mathrm{O}-123.3 \mathrm{mg} / \mathrm{L} \mathrm{NaHCO}_{3}-64.8 \mathrm{mg} / \mathrm{L}$, $\mathrm{KCl}-5.8 \mathrm{mg} / \mathrm{L}$.

F. Statistical analysis. $\mathrm{EC}_{50 \mathrm{FI}}$ obtained from the feeding-suppression assays for 4 and $5 \mathrm{~h}$ were compared with $\mathrm{EC}_{50}$ immobilization test using analysis of variance to discover significant differences between pairwise groups.

\section{RESULT AND DISCUSSION}

In feeding experiment it would rather to bring some feeding conditions that closer to those conditions when daphnids were ingested the food having one nutritional value for them. It is known, that D. magna guts were nearly full of yeast or bacteria after 20 minutes and this period of time was required for beginning of defecation of these digestive cells (Taylor et. al, 1998).

Acs et al., 2009 found the probably exposure of fluorescence substance that is not digestible and inert or more than 20 min may decrease food intake and affect filtration rate. In our study measured the fluorescence of microbeads $(\mathrm{d}=0.2 \mu \mathrm{m})$, though the ingested microbeds shown the beginning of defecation after only 5 minutes, it was observed the max fluorescence for 20-25 minutes of exposure in microbeds solution..

Kovacs et al., 2012 using developed by Acs et al., 2009 D. magna feeding depression bioassay (red polystyrene microsphere suspension with a particle size of $5 \mu \mathrm{m}$ ) found that after longer $(4 \mathrm{~h})$ exposure, the feeding inhibition test proved to have similar sensitivity in comparison with the standard immobility test and also gave valid results according to the ISO 6341 standard (USEPA, 1985).

The result of our previous study (Kamay et al., 2011; Ginatullina et al., 2013; Kamaya et al., 2016) to measure of D. magna feeding suppression when we used as fluorescence substance showed the best result for fluorescence measurement and linear regression between feeding rate and $\log$ of toxicant concentration for $6 \mathrm{~h}$ than for $3 \mathrm{~h}$ simultaneously exposure in toxicant and food.

In this study we carried out several acute feeding experiments for exposure 1, 2, 3, 4 and 5 hour and the 24 hour' lethality test using the pretty similar dilution serial of potassium dichromate concentration for each bioassay.

In Table 3 shown the calculated of immobilization and feeding suppression test endpoints after exposure in standard toxicant. Data expressed as mean of three replicates

Table 3 The $\mathrm{EC}_{50}$ and $\mathrm{EC}_{50 \mathrm{FI}}$ endpoints of $\mathrm{K}_{2} \mathrm{Cr}_{2} \mathrm{O}_{7}$

\begin{tabular}{|c|c|}
\hline $\begin{array}{c}\text { Exposure time in toxicant } \\
\text { solution }\end{array}$ & $\begin{array}{c}\text { Mean of endpoints } \pm \\
\text { SD }\end{array}$ \\
\hline $1 \mathrm{~h}$ & $2.03 \pm 0.35$ \\
\hline $2 \mathrm{~h}$ & $2.09 \pm 0.41$ \\
\hline $3 \mathrm{~h}$ & $1.7 \pm 0.38$ \\
\hline $4 \mathrm{~h}$ & $1.44 \pm 0.04$ \\
\hline $5 \mathrm{~h}$ & $1.08 \pm 0.19$ \\
\hline $24 \mathrm{~h}$ & $1.16 \pm 0.15$ \\
\hline
\end{tabular}

We compared calculated feeding EC $_{50 \mathrm{FI}}$ endpoints with $24 \mathrm{~h}$ immobilization endpoint. The endpoints for 1, 2, 3, 4 and 5 hours feeding bioassay were not significantly different from EC $_{50}$ of 24 hour immobilization test. However, the EC $_{50 \mathrm{FI}}$ for 4 and 5 hours feeding bioassay shown less difference compare to value of $24 \mathrm{~h}$ test than data for 1, 2 and 3 hour's bioassay.

\section{CONCLUSIONS}

Generally, for 1, 2, 3, 4 and 5 hours feeding suppression bioassay $\mathrm{EC}_{50 \mathrm{FI}}$ endpoints were not different significantly from $\mathrm{EC}_{50}$ of immobilization concentration. However, for 1, 2 and 3 hours bioassay the standard deviations have relatively higher value than for 4 and 5 hours test. As a result of the comparative study, 4 hours exposure for feeding suppression bioassay definite as the most suitable time period for further experiments on feeding suppression test.

\section{REFERENCES}

1. Ács A., Kováts N. and Refaey M., 2009. Novel Daphnia test for detecting chemical pollution. In: Simeonoy L.I. and Hassanien M.A. (eds.), Exposure and risk assessment of chemical pollution Contemporary Methodology, Springer, 439-444.

2. De Coen W.M. and Janssen C.R., 1998. The use of biomarkers in Daphnia magna toxicity testing. I. The digestive physiology of daphnids exposed to toxic stress. Hydrobiologia, 367, 199-209.

3. De Coen W.M., Vangheluwe M.L. and Janssen C.R., 1998. The use of biomarkers in Daphnia magna toxicity testing. III. Rapid toxicity testing of pure chemicals and sediment pore waters using ingestion and digestive enzyme activity. Chemosphere, 37, 2677-2694.

4. Ferrando M.D. and Andreu E., 1993. Feeding behavior as an index of copper stress in Daphnia magna and Brachionus calyciflorus. Comp. Biochem. Physiol., C, 106, 327-331.

5. Fernandez-Cassalderrey A., Ferrando M.D. and Andreu-Moliner E., 1994. Effects of sublethal concentrations of pesticides on the feeding behaviour of Daphnia magna. Ecotoxicol. Environ. Saf., 27, 82-89.

6. Ginatullina, E., Chiken, A., Atwell, L., Kamaya, M. (2013): Engineering of a new acute bioassay design based on feeding behavior of D. magna with fluorescent microbeads. International Journal of Emerging Technology and Advanced Engineering, 3(11): 671-677.

7. Kamaya, M., Ginatullina, E., Wani, N. (2016): Fluorescence intensity assay based on suppression of Daphnia magna feeding on dry algae (Chlorella and Spirullina). International Journal of Emerging Technology and Advanced Engineering, 6 (12): 41-46.

8. Kamaya M., Sonamoto, M., Nagashima, K., Ginatullina E. (2011): A simple bioassay using fluorescent microbeads and Daphnia magna. Journal of Environmental Science and Engineering: 5(12):1613-1617.

9. Lürling M., de Lange H.J. and Peeters E.T.H.M., 2011. Effects of an anionic surfactant (FFD-6) on the energy and information flow between a primary producer (Scenedesmus obliquus) and a consumer (Daphnia magna). Ecotoxicology, 20, 1881-1889.

10. Tarazona, J.V., 2014. Pollution, Water.Encyclopedia of Toxicology.Thirdedition.Vol. 1. Elsevier, pp: 1024-1027.

11. Braginsky, L.P., 2000. Methodological aspects of toxicology bioassay on Daphnia magna and other cladocerans (critical review).Hydrobiological Journal, 36(5): 50-70.

12. Kovacs A., Abdel-Hameid N.A., Acs A., Kovats N., 2012 A novel protocol for assessing aquatic pollution based on the feeding inhibition of Daphnia magna. Journal of Knowledge and management of aquatic ecosystem, 404, 07.

13. Taylor G, Baird DJ, SoaresAMVM(1998). Surface binding of contaminants by algae: consequences for lethal toxicity and feeding to Daphnia magna Straus. Environmental Toxicology and Chemistry 17 (3): 412-419.

14. USEPA(1985). U.S., Short-term Methods for Estimating the Chronic Toxicity of Effluents and Receiving Waters to Freshwater Organisms.Fourth ed.; Washington, 2002; p 334.

15. J.I.S. (1992). Japanese Industrial Standard № K 0229/1992 on testing ethods for determination of the inhibition of mobility of Daphnia by chemicals.

Blue Eyes Intelligence Engineering's \& Sciences Publication (C) Copyright: All rights reserved. 
The Fluorescent Measurement in D. magna feeding suppression bioassay during the pre-exposure in potassium dichromate for 1, 2, 3,4 and 5 hours

\section{AUTHORS PROFILE}

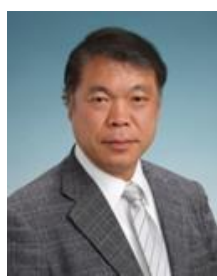

Kamaya Minori, Head of Environmental Analytical Chemistry Laboratory, Kogakuin University, Tokyo. Scientific fields are: development of simple and prompt analytical method for surfactants; developing ion chromatography' method for anions; design of spectrophotometric determination of hydrogen molecules; simple determination method for removal of reactive silicate using hydrotalcite substance; developing an acute toxicity test using $D$. magna filtration ability and fluorescent spectrophotometry. There are 110 publications.

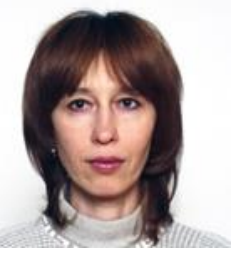

Ginatullina Elena, From 2019 head of Laboratory Hydrobiology at Institute Zoology of Uzbekistan Academy of Science. Scientific fields are: water quality assessment of o artificial reservoir using the chemical and hydro biological (biodiversity) indexes; study of bio diversity of phytoplankton and zooplankton in saline lakes; aquatic ecology and water chemistry; assessment of toxicity the chemical substances using Cladocera specimen. Doctoral thesis: "Zooplankton communities as indicator of water quality of drainage saline lakes of the Aral Sea west-south region in Uzbekistan".

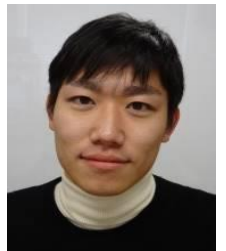

Yamagata Kohei, the second course master student of Environmental Analytical Chemistry Laboratory, Kogakuin University, Tokyo. During two years he is carrying out experimental works an acute toxicity test of several heavy metals using $D$. magna filtration ability and fluorescent spectrophotometry. 